Pacific

Journal of

Mathematics

TESTING THE FUNCTIONAL EQUATION OF A HIGH-DEGREE EULER PRODUCT

DAVID W. FARMER, NATHAN C. RYAN AND RALF SCHMIDT

Volume $253 \quad$ No. 2

October 2011 


\title{
TESTING THE FUNCTIONAL EQUATION OF A HIGH-DEGREE EULER PRODUCT
}

\author{
DAVID W. FARMER, NATHAN C. RYAN AND RALF SCHMIDT
}

\begin{abstract}
We study the L-functions associated to Siegel modular forms - equivalently, automorphic representations of $\operatorname{GSp}\left(4, \mathbb{A}_{\mathbb{Q}}\right)$ - both theoretically and numerically. For the L-functions of degrees 10, 14, and 16 we perform representation theoretic calculations to cast the Langlands L-function in classical terms. We develop a precise notion of what it means to test a conjectured functional equation for an L-function, and we apply this to the degree-10 adjoint L-function associated to a Siegel modular form.
\end{abstract}

\section{Introduction}

L-functions are special functions that arise in representation theory and in several areas of number theory. From the viewpoint of analytic number theory, L-functions are Dirichlet series with a functional equation and an Euler product. From the point of view of representation theory, L-functions arise from automorphic representations of a reductive group over the adeles of a number field.

The two points of view offer distinct benefits. Representation theory, via the Langlands program [Gelbart 1984], provides a framework for understanding how L-functions arise, as well as the connections between various mathematical objects. L-functions considered as objects of analytic number theory are suitable for concrete exploration and testing of conjectures; for example, they can be evaluated on a computer to check the Riemann hypothesis. Unfortunately, it can be quite difficult to translate Langlands L-functions into this setting, which limits the ability to do explicit calculations and test conjectures. In this paper we make such a translation and perform computer calculations with the results: for a particular Siegel modular form $F$, we calculate factors $L_{p}(s, F, \rho)$ and $\varepsilon_{p}(s, F, \rho)(p \leq \infty)$ for five choices of $\rho$ (dimensions $4,5,10,14,16$ ) using the Langlands parametrization of the discrete series. Using these calculations we provide numerical evidence that the L-function of degree 10 satisfies a functional equation.

The L-functions we consider here arise (in the classical setting) from holomorphic Siegel modular forms on $\operatorname{Sp}(4, \mathbb{Z})$, see Section 2. The same L-functions arise

Keywords: Siegel modular forms, L-functions. 
from automorphic representations of $\operatorname{PGSp}(4, \mathbb{A}$ ), see Section 3 (as in [Asgari and Schmidt 2001], at the archimedean place such an automorphic representation is a holomorphic discrete series representation and at the nonarchimedean places it is a spherical principal series representation). The Langlands program predicts the existence of an infinite list of L-functions associated to a Siegel modular form. In our particular case the first two L-functions are known as the spinor and the standard L-function, and have degree 4 and 5, respectively. Due to Andrianov [1974], Shimura [1994], Böcherer [1985] and others, these L-functions are fairly well-understood; for Siegel modular forms on $\operatorname{Sp}(4, \mathbb{Z})$, they are known to be entire functions that satisfy a functional equation.

The next case is the adjoint $\mathrm{L}$-function, which has degree 10. It has not been shown that this L-function is entire and satisfies a functional equation. (The automorphic representations associated to holomorphic Siegel modular forms are not generic, so a technique as in [Ginzburg 1996] is not applicable.) Providing evidence for the conjectured functional equation, via a computer calculation, is one of the goals of this paper. This is made precise in Theorem 4.2, which gives a test for the functional equation and quantifies the probability that the test could yield a false positive.

A substantial part of this paper is a translation from the perspective of representation theory to the viewpoint of analytic number theory. Selberg [1992] gave a set of axioms for what is now called the "Selberg class" of L-functions. We will call L-functions in this class "Selberg L-functions," and are to be compared with Langlands L-functions - those that arise from automorphic representations. It is a standard conjecture that all Selberg L-functions are Langlands L-functions and that all primitive Langlands L-functions are Selberg L-functions. In this paper we translate Langlands L-functions into Selberg L-functions. We now describe Selberg L-functions in more detail.

A Selberg L-function $L(s)$ is given by a Dirichlet series

$$
L(s)=\sum_{n=1}^{\infty} \frac{a_{n}}{n^{s}}
$$

where $a_{1}=1$ and the series converges in some half-plane. We assume a Ramanujan bound on the coefficients: $a_{n}=O\left(n^{\varepsilon}\right)$ for any $\varepsilon>0$. Moreover, it has a meromorphic continuation to the whole complex plane with at most finitely many poles, all of which in the half-plane $\Re(s)>0$ are on the line $\Re(s)=1$. L $(s)$ can be written as an Euler product

$$
L(s)=\prod_{p} L_{p}\left(p^{-s}\right)^{-1},
$$

where the product is over the primes and $L_{p}$ is a polynomial with $L_{p}(0)=1$. Additionally, there exist $Q>0$, positive real numbers $\kappa_{1}, \ldots, \kappa_{n}$, and complex 
numbers with nonnegative real part $\mu_{1}, \ldots, \mu_{n}$, such that

$$
\Lambda(s):=Q^{s} \prod \Gamma\left(\kappa_{j} s+\mu_{j}\right) \cdot L(s)
$$

is meromorphic with poles only arising from the poles of $L(s)$ and satisfies the functional equation $\Lambda(s)=\varepsilon \overline{\Lambda(1-\bar{s})}$ where $|\varepsilon|=1$. The number $d=2 \sum \kappa_{j}$ is the degree of the L-function.

Indeed, for the L-functions considered here there do not exist results in the literature which would allow a nonexpert to translate the L-function data from representation theoretic language into a form involving a Dirichlet series. Thus, we give a brief introduction to the aspects of the Langlands program that are relevant to our calculations, and describe how to translate the Langlands L-functions we consider here into Selberg L-functions. The results of those calculations are summarized in Proposition 2.1.

In Section 2 we describe the Siegel modular forms which give rise to the Lfunctions considered here, and we describe these L-functions in the classical language. In Section 3 we describe Langlands L-functions and how to translate the degree-10 L-function considered here into a form which can be evaluated on a computer. In Section 4 we provide evidence for the conjectured functional equations and also briefly address the problem of accurately evaluating L-functions for which only a few of the local factors in the Euler product are known. We also provide criteria to measure the strength of the evidence.

\section{Siegel modular forms and their L-functions}

We recall the definition and main properties of Siegel modular forms on $\operatorname{Sp}(2 n, \mathbb{Z})$ and we describe the two simplest L-functions associated to them.

Siegel modular forms. Let $0_{n}$ be the zero matrix,

$$
E_{n}:=\left({ }_{1} \cdot{ }^{1}\right) \quad \text { and } \quad J_{n}:=\left(\begin{array}{rr}
0_{n} & E_{n} \\
-E_{n} & 0_{n}
\end{array}\right) .
$$

Denote the group of symplectic similitudes by

$$
\left.\mathrm{GSp}^{+}(2 n, \mathbb{R}):=\left\{\alpha \in \mathrm{GL}(2 n, \mathbb{R}):^{t} \alpha J_{n} \alpha=r(\alpha) J_{n}, r(\alpha) \in \mathbb{R}, r(\alpha)>0\right)\right\},
$$

where $r(\alpha)$ is called the similitude of $\alpha$. Define the Siegel modular group of genus $n$ by $\operatorname{Sp}(2 n, \mathbb{Z}):=\left\{\gamma \in \mathrm{GSp}^{+}(2 n, \mathbb{R}) \cap M(2 n, \mathbb{Z}): r(\gamma)=1\right\}$. Let

$$
\mathscr{H}^{n}:=\left\{Z=X+i Y: X, Y \in M(n, \mathbb{R}),{ }^{t} Z=Z, Y>0\right\}
$$

denote the Siegel upper half space, that is, symmetric matrices in $M(n, \mathbb{C})$ with positive definite imaginary part. 
Recall, a holomorphic function $F: \mathscr{H}^{n} \rightarrow \mathbb{C}$ is a Siegel modular form of genus $n$ and weight $k$ if for all $\alpha=\left(\begin{array}{ll}A & B \\ C & D\end{array}\right) \in \operatorname{Sp}(2 n, \mathbb{Z})$ it satisfies the transformation property

$$
F(Z)=\left(\left.F\right|_{k} \alpha\right)(Z):=r(\alpha)^{n k-n(n+1) / 2} \operatorname{det}(C Z+D)^{-k} F\left((A Z+B)(C Z+D)^{-1}\right) .
$$

If $n=1$ then $F$ must satisfy an additional growth condition.

We denote the space of weight $k$ genus 2 Siegel modular forms by $M_{k}(\operatorname{Sp}(4, \mathbb{Z}))$. In genus 2, we can express the expansion of a Siegel cusp form as

$$
F(Z)=\sum_{\substack{r, n, m \in \mathbf{Z} \\ r^{2}-4 m n<0 \\ n, m \geq 0}} a_{F}(n, r, m) q^{n} \zeta^{r} q^{\prime n}
$$

where $[n, r, m]$ is the positive definite binary quadratic form $n X^{2}+r X Y+m Y^{2}$ of discriminant $r^{2}-4 m n$ and $q=e^{2 \pi i z}\left(z \in \mathscr{H}^{1}\right), q^{\prime}=e^{2 \pi i \omega}\left(\omega \in \mathscr{H}^{1}\right)$, and $\zeta=e^{2 \pi i \tau}(\tau \in \mathbb{C})$. In particular, we are examining L-functions associated to modular forms not in the Maass space, i.e., not in the image of the Saito-Kurokawa lift, i.e., whose L-functions are primitive (at least conjecturally). The first such form occurs in weight $k=20$ and is computed in [Skoruppa 1992].

There is a theory of Hecke operators acting on the space of Siegel modular forms; we denote the $n$-th Hecke operator by $T(n)$. The Hecke eigenvalues for a Siegel modular form can be computed explicitly from its Fourier coefficients, but this is computationally expensive. Let $F$ be a Hecke eigenform; i.e., suppose that for each $n$ there exists a $\lambda_{F}(n)$ such that

$$
F \mid T(n)=\lambda_{F}(n) F .
$$

For the weight 20 Siegel cusp form $F$ that is not a Saito-Kurokawa lift, we will use $\lambda_{F}(p)$ and $\lambda_{F}\left(p^{2}\right)$ for $p \leq 79$ in our experiments. These data are computed in [Kohnen and Kuss 2002].

L-functions associated to Siegel modular forms. There are two well-known Lfunctions attached to Siegel modular forms on $\mathrm{Sp}(4, \mathbb{Z})$, called the spinor L-function and the standard L-function. They have been studied by Andrianov [1974], Shimura [1994], Böcherer [1985] and others. Formulas for those L-functions in the genus 2 case are given in Proposition 2.1.

Given a genus-2 eigenform $F$ and a prime $p$, there is a triple $\left(\alpha_{0, p}, \alpha_{1, p}, \alpha_{2, p}\right)$ of nonzero complex numbers - it is in these terms that our L-functions are expressed. The entries of the triple are called the Satake parameters of $F$.

In genus 2 it is rather straightforward to compute the Satake parameters of a form, given the Hecke eigenvalues of the form. By using an explicit description of the Satake isomorphism, as found, for example, in [Ryan and Shemanske 2008], 
one can write the Euler factor of the spinor L-function as a polynomial whose coefficients are in terms of $\lambda_{F}(p)$ and $\lambda_{F}\left(p^{2}\right)$. To compute the Satake parameters, one finds the roots of this polynomial.

We rescale the Satake parameters to have the normalization

$$
\left|\alpha_{j}\right|=1, \quad \alpha_{0}^{2} \alpha_{1} \alpha_{2}=1,
$$

which is possible since the Ramanujan bound is a theorem for Siegel modular forms [Weissauer 2009]. This corresponds to a simple change of variable in the L-function, so that all our L-functions satisfy a functional equation in the standard form $s \leftrightarrow 1-s$.

In Section 3 we describe the procedure for determining the L-functions associated to an automorphic representation, and give reasonably complete details for the spinor, standard, and adjoint L-functions of genus 2 Siegel modular forms. The results of those calculations are summarized in the following proposition. For completeness we also include the results of similar computations carried out for two more L-functions: ones associated to a specific 14-dimensional representation and 16-dimensional representation, denoted $\rho_{14}$ and $\rho_{16}$.

Proposition 2.1. Suppose $F \in M_{k}(\mathrm{Sp}(4, \mathbb{Z}))$ is a Hecke eigenform. Let $\alpha_{0, p}, \alpha_{1, p}$, $\alpha_{2, p}$ be the Satake parameters of $F$ for the prime $p$, where we suppress the dependence on $p$ in the formulas below. For $\rho \in\left\{\operatorname{spin}\right.$, stan, adj, $\left.\rho_{14}, \rho_{16}\right\}$ we have the L-functions

$$
L(s, F, \rho):=\prod_{p \text { prime }} Q_{p}\left(p^{-s}, F, \rho\right)^{-1}
$$

where

$Q_{p}(X, F$, spin $):=\left(1-\alpha_{0} X\right)\left(1-\alpha_{0} \alpha_{1} X\right)\left(1-\alpha_{0} \alpha_{2} X\right)\left(1-\alpha_{0} \alpha_{1} \alpha_{2} X\right)$, $Q_{p}(X, F, \operatorname{stan}):=(1-X)\left(1-\alpha_{1} X\right)\left(1-\alpha_{1}^{-1} X\right)\left(1-\alpha_{2} X\right)\left(1-\alpha_{2}^{-1} X\right)$,

$Q_{p}(X, F, \operatorname{adj}):=(1-X)^{2}\left(1-\alpha_{1} X\right)\left(1-\alpha_{1}^{-1} X\right)\left(1-\alpha_{2} X\right)\left(1-\alpha_{2}^{-1} X\right)$

$$
\left(1-\alpha_{1} \alpha_{2} X\right)\left(1-\alpha_{1}^{-1} \alpha_{2} X\right)\left(1-\alpha_{1} \alpha_{2}^{-1} X\right)\left(1-\alpha_{1}^{-1} \alpha_{2}^{-1} X\right) \text {, }
$$

$Q_{p}\left(X, F, \rho_{14}\right):=(1-X)^{2}\left(1-\alpha_{1} X\right)\left(1-\alpha_{2} X\right)\left(1-\alpha_{1}^{-1} X\right)\left(1-\alpha_{2}^{-1} X\right)$

$$
\begin{aligned}
& \left(1-\alpha_{1}^{2} X\right)\left(1-\alpha_{2}^{2} X\right)\left(1-\alpha_{1}^{-2} X\right)\left(1-\alpha_{2}^{-2} X\right) \\
& \left(1-\alpha_{1} \alpha_{2} X\right)\left(1-\alpha_{1} \alpha_{2}^{-1} X\right)\left(1-\alpha_{1}^{-1} \alpha_{2} X\right)\left(1-\alpha_{1}^{-1} \alpha_{2}^{-1} X\right),
\end{aligned}
$$

$Q_{p}\left(X, F, \rho_{16}\right):=\left(1-\alpha_{0} X\right)^{2}\left(1-\alpha_{0} \alpha_{1} X\right)^{2}\left(1-\alpha_{0} \alpha_{2} X\right)^{2}\left(1-\alpha_{0} \alpha_{1} \alpha_{2} X\right)^{2}$

$$
\begin{aligned}
& \left(1-\alpha_{0} \alpha_{1}^{-1} X\right)\left(1-\alpha_{0} \alpha_{2}^{-1} X\right)\left(1-\alpha_{0} \alpha_{1}^{2} X\right)\left(1-\alpha_{0} \alpha_{2}^{2} X\right) \\
& \left(1-\alpha_{0} \alpha_{1} \alpha_{2}^{-1} X\right)\left(1-\alpha_{0} \alpha_{1}^{-1} \alpha_{2} X\right)\left(1-\alpha_{0} \alpha_{1}^{2} \alpha_{2} X\right)\left(1-\alpha_{0} \alpha_{1} \alpha_{2}^{2} X\right),
\end{aligned}
$$


give the L-series of, respectively, the spinor, standard, adjoint, degree-14 and degree-16 L-functions. These L-functions satisfy the following functional equations (conjecturally in the case of adjoint, degree-14 and degree-16 L-functions):

$$
\begin{aligned}
\Lambda(s, F, \text { spin }):= & \Gamma_{\mathbb{C}}\left(s+\frac{1}{2}\right) \Gamma_{\mathbb{C}}\left(s+k-\frac{3}{2}\right) L(s, F, \text { spin }) \\
= & (-1)^{k} \Lambda(1-s, F, \text { spin }), \\
\Lambda(s, F, \operatorname{stan}):= & \Gamma_{\mathbb{R}}(s) \Gamma_{\mathbb{C}}(s+k-2) \Gamma_{\mathbb{C}}(s+k-1) L(s, F, \text { stan }) \\
= & \Lambda(1-s, F, \operatorname{stan}), \\
\Lambda(s, F, a d j):= & \Gamma_{\mathbb{R}}(s+1)^{2} \Gamma_{\mathbb{C}}(s+1) \\
& \times \Gamma_{\mathbb{C}}(s+k-2) \Gamma_{\mathbb{C}}(s+k-1) \Gamma_{\mathbb{C}}(s+2 k-3) L(s, F, \operatorname{adj}) \\
= & \Lambda(1-s, F, \operatorname{adj}), \\
\Lambda\left(s, F, \rho_{14}\right):= & \Gamma_{\mathbb{R}}(s)^{2} \Gamma_{\mathbb{C}}(s+1) \Gamma_{\mathbb{C}}(s+k-2) \Gamma_{\mathbb{C}}(s+k-1) \\
& \quad \times \Gamma_{\mathbb{C}}(s+2 k-4) \Gamma_{\mathbb{C}}(s+2 k-3) \Gamma_{\mathbb{C}}(s+2 k-2) L\left(s, F, \rho_{14}\right) \\
= & \Lambda\left(1-s, F, \rho_{14}\right), \text { and } \\
\Lambda\left(s, F, \rho_{16}\right):= & \Gamma_{\mathbb{C}}\left(s+\frac{1}{2}\right)^{2} \Gamma_{\mathbb{C}}\left(s+k-\frac{5}{2}\right) \Gamma_{\mathbb{C}}\left(s+k-\frac{3}{2}\right)^{2} \Gamma_{\mathbb{C}}\left(s+k-\frac{1}{2}\right) \\
& \quad \times \Gamma_{\mathbb{C}}\left(s+2 k-\frac{5}{2}\right) \Gamma_{\mathbb{C}}\left(s+2 k-\frac{7}{2}\right) L\left(s, F, \rho_{16}\right) \\
= & -\Lambda\left(1-s, F, \rho_{16}\right) .
\end{aligned}
$$

In (2-2), we use the normalized $\Gamma$-functions

$$
\Gamma_{\mathbb{R}}(s):=\pi^{-s / 2} \Gamma(s) \quad \text { and } \quad \Gamma_{\mathbb{C}}(s):=2(2 \pi)^{-s} \Gamma(s) .
$$

\section{Langlands L-functions}

The Euler products in the previous section arise as Langlands $L$-functions attached to automorphic representations of $\mathrm{GSp}(4, \mathbb{A})$ generated by the Siegel modular form $F$; see [Asgari and Schmidt 2001]. In general, this procedure involves the local Langlands correspondence, which is now a theorem for GSp(4); see [Gan and Takeda 2010]. However, since we are only interested in full level Siegel modular forms, the mechanism simplifies considerably. We shall briefly describe how to obtain the local factors in the nonarchimedean and the archimedean case. In doing this, we indicate how one proves Proposition 2.1 and results like it.

The nonarchimedean factors. Let $\alpha_{0}, \alpha_{1}, \alpha_{2}$ be the Satake parameters of $F$ at a finite place $p$, normalized as above, so that $\alpha_{0}^{2} \alpha_{1} \alpha_{2}=1$. They determine a semisimple conjugacy class in the dual group $\operatorname{Sp}(4, \mathbb{C})$, represented by the diagonal matrix

$$
A_{\pi_{p}}=\operatorname{Diag}\left(\alpha_{0}, \alpha_{0} \alpha_{1}, \alpha_{0} \alpha_{2}, \alpha_{0} \alpha_{1} \alpha_{2}\right) .
$$


(One has to carefully go through the definitions of the local Langlands correspondence to see this; see [Roberts and Schmidt 2007, §§2.3 and 2.4].) Let $\rho: \operatorname{Sp}(4, \mathbb{C}) \rightarrow \operatorname{GL}(n, \mathbb{C})$ be a finite-dimensional representation of the dual group. The local L-factor attached to the data $\alpha_{0}, \alpha_{1}, \alpha_{2}$ and $\rho$ is given by

$$
L_{p}(s, F, \rho)=\frac{1}{\operatorname{det}\left(1-p^{-s} \rho\left(A_{\pi_{p}}\right)\right)} .
$$

The three smallest nontrivial irreducible representations of $\operatorname{Sp}(4, \mathbb{C})$ are the fourdimensional "spin" representation (which is simply the inclusion of $\operatorname{Sp}(4, \mathbb{C}$ ) into $\mathrm{GL}(4, \mathbb{C})$ ), the five-dimensional "standard" representation (described explicitly in [Roberts and Schmidt 2007, Section A.7]), and the ten-dimensional adjoint representation adj on the Lie algebra $\mathfrak{s p}(4, \mathbb{C})$. Calculations show that the resulting L-factors are given as

$$
\begin{aligned}
L_{p}(s, F, \text { spin }) & =Q_{p}\left(p^{-s}, F, \text { spin }\right), \\
L_{p}(s, F, \text { stan }) & =Q_{p}\left(p^{-s}, F, \text { stan }\right), \\
L_{p}(s, F, \text { adj }) & =Q_{p}\left(p^{-s}, F, \text { adj }\right),
\end{aligned}
$$

with the factors $Q_{p}$ as in the previous section. There are also corresponding local $\varepsilon$-factors $\varepsilon_{p}(s, F, \rho)$, which for unramified representations are all constantly 1 .

The archimedean factors. The real Weil group $W_{\mathbb{R}}$ is given by $W_{\mathbb{R}}=\mathbb{C}^{\times} \sqcup j \mathbb{C}^{\times}$ with the rules $j^{2}=-1$ and $j c j^{-1}=\bar{c}$; see [Tate 1979, (1.4.3)]. The commutator subgroup is $S^{1} \subset \mathbb{C}^{\times}$, the set of complex numbers with absolute value 1 . There is a reciprocity law isomorphism

$$
\begin{aligned}
r_{\mathbb{R}}: \mathbb{R}^{\times} & \stackrel{\sim}{\longrightarrow} W_{\mathbb{R}}^{\mathrm{ab}}, \\
-1 & \longmapsto j S^{1}, \\
\mathbb{R}_{>0} \ni x & \longmapsto \sqrt{x} S^{1} .
\end{aligned}
$$

Let $|\cdot|$ be the usual absolute value on $\mathbb{R}$, and let $\|\cdot\|$ be the character of $W_{\mathbb{R}}$ defined by the commutativity of the diagram

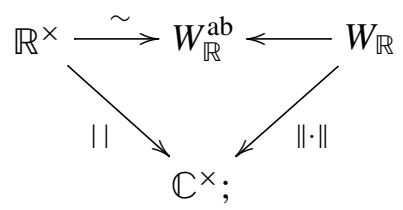

see [Tate $1979,(1.4 .5)]$. Hence, $\|z\|=|z|^{2}$ for $z \in \mathbb{C}^{\times}$, where $|\cdot|$ denotes the usual absolute value on $\mathbb{C}$. The character $\|\cdot\|^{s}$ is denoted by $\omega_{s}$, for a complex number $s$, as in [Tate $1979,(2.2)]$. There are L- and $\varepsilon$-factors attached to characters of $\mathbb{R}^{\times}$; see 
[Tate 1979, (3.1)]. The correspondence between characters of $W_{\mathbb{R}}$ and characters of $\mathbb{R}^{\times}$, and the associated L- and $\varepsilon$-factors, is given in the following table.

\begin{tabular}{|c|c|c|c|}
\hline char. of $W_{\mathbb{R}}$ & char. of $\mathbb{R}^{\times}$ & L-factor & $\varepsilon$-factor \\
\hline \hline$\varphi_{+, t}: z \mapsto|z|^{t}, j \mapsto 1$ & $x \mapsto|x|^{t}$ & $\Gamma_{\mathbb{R}}(s+t)$ & 1 \\
\hline$\varphi_{-, t}: z \mapsto|z|^{t}, j \mapsto-1$ & $x \mapsto \operatorname{sgn}(x)|x|^{t}$ & $\Gamma_{\mathbb{R}}(s+t+1)$ & $i$ \\
\hline
\end{tabular}

Besides one-dimensional representations, the only other irreducible representations of $W_{\mathbb{R}}$ are two-dimensional and indexed by pairs $(\ell, t)$, where $\ell$ is a positive integer and $t \in \mathbb{C}$. The representation attached to $(\ell, t)$ is $\varphi_{\ell, t}$, given by

$$
\varphi_{\ell, t}: r e^{i \theta} \longmapsto\left[\begin{array}{cc}
r^{t} e^{i \ell \theta} & \\
& r^{t} e^{-i \ell \theta}
\end{array}\right], \quad j \longmapsto\left[\begin{array}{ll}
(-1)^{\ell} \\
1
\end{array}\right] .
$$

The associated L- and $\varepsilon$-factors are

$$
L\left(s, \varphi_{\ell, t}\right)=\Gamma_{\mathbb{C}}\left(s+t+\frac{\ell}{2}\right), \quad \varepsilon\left(s, \varphi_{\ell, t}\right)=i^{\ell+1} .
$$

Now, to a Siegel modular form $F$ of weight $k$ there is attached the four-dimensional representation of $W_{\mathbb{R}}$ given by

$$
\varphi_{(k-1, k-2)}:=\varphi_{1,0} \oplus \varphi_{2 k-3,0}
$$

(this is really the parameter of a holomorphic discrete series representation with Harish-Chandra parameter $(k-1, k-2)$; see [Borel 1979]). The image of this parameter can be conjugated into the dual group $\operatorname{Sp}(4, \mathbb{C})$. Given a finite-dimensional representation $\rho: \operatorname{Sp}(4, \mathbb{C}) \rightarrow \operatorname{GL}(n, \mathbb{C})$, we compose $\rho$ with the representation (3-8) and obtain an $n$-dimensional representation of $W_{\mathbb{R}}$. By [Knapp 1994], this representation can be decomposed into one- and two-dimensional irreducibles. The product of the L-factors (resp. $\varepsilon$-factors) attached to these irreducibles is by definition $L_{\infty}(s, F, \rho)\left(\right.$ resp. $\left.\varepsilon_{\infty}(s, F, \rho)\right)$. Calculations show that

$$
\begin{aligned}
L_{\infty}(s, F, \operatorname{spin}) & =\Gamma_{\mathbb{C}}\left(s+\frac{1}{2}\right) \Gamma_{\mathbb{C}}\left(s+k-\frac{3}{2}\right), \\
L_{\infty}(s, F, \operatorname{stan}) & =\Gamma_{\mathbb{R}}(s) \Gamma_{\mathbb{C}}(s+k-1) \Gamma_{\mathbb{C}}(s+k-2), \\
L_{\infty}(s, F, \operatorname{adj}) & =\Gamma_{\mathbb{R}}(s+1)^{2} \Gamma_{\mathbb{C}}(s+1) \Gamma_{\mathbb{C}}(s+k-2) \Gamma_{\mathbb{C}}(s+k-1) \Gamma_{\mathbb{C}}(s+2 k-3),
\end{aligned}
$$

and that

$$
\begin{aligned}
\varepsilon_{\infty}(s, F, \operatorname{spin}) & =(-1)^{k}, \\
\varepsilon_{\infty}(s, F, \operatorname{stan}) & =1, \\
\varepsilon_{\infty}(s, F, \operatorname{adj}) & =1 .
\end{aligned}
$$

We see that the archimedean L-factors coincide with the $\Gamma$-factors in (2-2). 
The global L-function. Having defined all local factors, the global L-function attached to $F$ and a finite-dimensional representation $\rho: \operatorname{Sp}(4, \mathbb{C}) \rightarrow \operatorname{GL}(n, \mathbb{C})$ is given by

$$
\Lambda(s, F, \rho)=\prod_{p \leq \infty} L_{p}(s, F, \rho) .
$$

Up to a constant, this definition coincides with the Euler products defined in (2-1). By general conjectures, the global L-function, which is convergent in some right halfplane, should have meromorphic continuation to all of $\mathbb{C}$ and satisfy the functional equation $^{1}$

$$
\Lambda(1-s, F, \rho)=\varepsilon(s, F, \rho) \Lambda(s, F, \rho), \quad \text { where } \varepsilon(s, F, \rho)=\prod_{p \leq \infty} \varepsilon_{p}(s, F, \rho)
$$

Note that in our case $\varepsilon(s, F, \rho)=\varepsilon_{\infty}(s, F, \rho)$. Hence, the functional equations (2-2) are all special cases of the general conjectured functional Equation (3-9).

\section{Checking the functional equation}

As mentioned in the introduction, the degree-10 adjoint L-function associated to a Siegel modular form has not been proven to satisfy a functional equation. We develop a method of checking a conjectured functional equation, and in Theorem 4.2 we provide a quantitative result that estimates the probability that this test could yield a false positive.

The main idea behind our method of testing a functional equation is that an L-function can be evaluated, at a given point and to a particular accuracy, using finitely many of its Dirichlet series coefficients. That evaluation makes fundamental use of the functional equation. Furthermore, this can be done in more than one way. The consistency of those calculations provides a check on the functional equation. We quantify the "probability" that the calculations are accidentally consistent by viewing the coefficients of the L-function as a random variable.

In the next section we describe the approximate functional equation and use it to evaluate an L-function. Subsequently (starting on page 360) we elaborate on the ideas from [Farmer et al. $\geq 2011$ ] to develop our method to check the functional equation for the degree-10 Euler product associated to a Siegel modular form.

Smoothed approximate functional equations. The material in this section is taken directly from [Rubinstein 2005, Section 3.2].

\footnotetext{
${ }^{1}$ The local $\varepsilon$-factors also depend on the choice of a local additive character. We are assuming a standard choice and hence do not reflect it in the notation. The global $\varepsilon$-factor is independent of the choice of global additive character.
} 
Let

$$
L(s)=\sum_{n=1}^{\infty} \frac{b_{n}}{n^{s}}
$$

be a Dirichlet series that converges absolutely in a half plane, $\Re(s)>\sigma_{1}$.

Let

$$
\Lambda(s)=Q^{s}\left(\prod_{j=1}^{a} \Gamma\left(\kappa_{j} s+\lambda_{j}\right)\right) L(s)
$$

with $Q, \kappa_{j} \in \mathbb{R}^{+}, \Re\left(\lambda_{j}\right) \geq 0$, and assume that:

(1) $\Lambda(s)$ has a meromorphic continuation to all of $\mathbb{C}$ with simple poles at $s_{1}, \ldots, s_{\ell}$ and corresponding residues $r_{1}, \ldots, r_{\ell}$.

(2) $\Lambda(s)=\varepsilon \overline{\Lambda(1-\bar{s})}$ for some $\varepsilon \in \mathbb{C},|\varepsilon|=1$.

(3) For any $\sigma_{2} \leq \sigma_{3}, L(\sigma+i t)=O\left(\exp t^{A}\right)$ for some $A>0$, as $|t| \rightarrow \infty$, $\sigma_{2} \leq \sigma \leq \sigma_{3}$, with $A$ and the constant in the $O$ notation depending on $\sigma_{2}$ and $\sigma_{3}$.

To obtain a smoothed approximate functional equation with desirable properties, Rubinstein [2005] introduces an auxiliary function. Let $g: \mathbb{C} \rightarrow \mathbb{C}$ be an entire function that, for fixed $s$, satisfies

$$
\left|\Lambda(z+s) g(z+s) z^{-1}\right| \rightarrow 0
$$

as $|\Im z| \rightarrow \infty$, in vertical strips, $-x_{0} \leq \Re z \leq x_{0}$. The smoothed approximate functional equation has the following form.

Theorem 4.1. For $s \notin\left\{s_{1}, \ldots, s_{\ell}\right\}$, and $L(s), g(s)$ as above,

$$
\Lambda(s)=g(s)^{-1}\left(\sum_{k=1}^{\ell} \frac{r_{k} g\left(s_{k}\right)}{s-s_{k}}+Q^{s} \sum_{n=1}^{\infty} \frac{b_{n}}{n^{s}} f_{1}(s, n)+\varepsilon Q^{1-s} \sum_{n=1}^{\infty} \frac{\bar{b}_{n}}{n^{1-s}} f_{2}(1-s, n)\right)
$$

where

$$
f_{1}(s, n):=\frac{1}{2 \pi i} \int_{\nu-i \infty}^{v+i \infty} \prod_{j=1}^{a} \Gamma\left(\kappa_{j}(z+s)+\lambda_{j}\right) z^{-1} g(s+z)(Q / n)^{z} d z,
$$

$$
f_{2}(1-s, n):=\frac{1}{2 \pi i} \int_{\nu-i \infty}^{v+i \infty} \prod_{j=1}^{a} \Gamma\left(\kappa_{j}(z+1-s)+\bar{\lambda}_{j}\right) z^{-1} g(s-z)(Q / n)^{z} d z
$$

with $v>\max \left\{0,-\Re\left(\lambda_{1} / \kappa_{1}+s\right), \ldots,-\Re\left(\lambda_{a} / \kappa_{a}+s\right)\right\}$. 
We assume $L(s)$ continues to an entire function, so the first sum in (4-3) does not appear. For fixed $Q, \kappa, \lambda, \varepsilon$, and sequence $b_{n}$, and $g(s)$ as described in the next section, the right side of (4-3) can be evaluated to high precision.

We illustrate the approximate functional equation with an example. Our examples use the genus 2 Siegel modular form which is the unique weight 20 eigenform, $F$, that is not a Saito-Kurokawa lift. We consider the degree-10 adjoint L-function associated to $F$, which we denote by $L_{F, 10}$. Conjecturally it satisfies the functional equation given in (2-2) in Proposition 2.1.

It is convenient to instead evaluate the "Hardy function" $Z_{F, 10}$ associated to $L_{F, 10}$. This function is defined by property that $Z_{F, 10}\left(\frac{1}{2}+i t\right)$ is real if $t$ is real, and

$$
\left|Z_{F, 10}\left(\frac{1}{2}+i t\right)\right|=\left|L_{F, 10}\left(\frac{1}{2}+i t\right)\right| .
$$

We use $L$ and $Z$ interchangeably in our discussions.

If we let $g(s)=1$ and $s=\frac{1}{2}+i$ then (4-3) gives

$$
\begin{aligned}
Z_{F, 10}\left(\frac{1}{2}+i\right)=1.15426 & +0.778012 b_{2}+0.50246 b_{3}+0.33776 b_{4} \\
+0.235813 b_{5}+\cdots & +0.0000142432 b_{82}+0.0000132692 b_{83}+\cdots \\
& +2.8771 \times 10^{-7} b_{149}+2.7402 \times 10^{-7} b_{150}+\cdots
\end{aligned}
$$

Here and throughout this paper, decimal values are truncations of the true values. The numerical calculations were done in Mathematica 7.0.1.0 on a Dell Inspiron 9300 laptop running Red Hat Linux.

If instead we let $g(s)=e^{-3 i s / 2}$ and keep $s=\frac{1}{2}+i$ then (4-3) gives

$$
\begin{aligned}
Z_{F, 10}\left(\frac{1}{2}+i\right)=1.3044 & +0.678149 b_{2}+0.314111 b_{3}+0.12853 b_{4} \\
+ & 0.0341584 b_{5}+\cdots+0.0000147237 b_{82}+0.0000123925 b_{83}+\cdots \\
& -1.28515 \times 10^{-6} b_{149}-1.22359 \times 10^{-6} b_{150}+\cdots
\end{aligned}
$$

To obtain a numerical value for $Z_{F, 10}\left(\frac{1}{2}+i\right)$ we need to know the coefficients $b_{n}$. The calculations in [Kohnen and Kuss 2002] provide the Satake parameters of $F$ for the primes $p \leq 79$, so Proposition 2.1 gives the local factors in the Euler product for $p \leq 79$. Expanding the product gives values for infinitely many $b_{n}$, including all $n \leq 82$, all composite $83<n \leq 79^{2}$, etc.

We evaluate (4-5) and (4-6) by computing 10,000 terms in the approximate functional equation. Using the known values of $b_{n}$ gives the main terms on the right side of (4-7). The error terms in (4-7) come from two sources. We use the Ramanujan bound to estimate the unknown $b_{n}$ for $n<10,000$ and directly determine their contribution to (4-5) and (4-6). (In cases where the Ramanujan bound is not known, one can usually make use of weaker results provided the bound is explicit.) To estimate the contributing of the terms with $n>10,000$ we combine the Ramanujan bound with Lemma 4.3. See page 364 for more details. 


\begin{tabular}{|c|c|c|c|c|c|}
\hline$\beta$ & $\frac{1}{2}$ & $\frac{1}{2}+i$ & $\frac{1}{2}+2 i$ & $\frac{1}{2}+3 i$ & $\frac{1}{2}+4 i$ \\
\hline 0 & $\begin{aligned} & 2.148764 \\
\pm & 0.00016\end{aligned}$ & $\begin{aligned} & 3.084662 \\
\pm & 0.00046\end{aligned}$ & $\begin{aligned} & 3.263120 \\
\pm & 0.0044\end{aligned}$ & $\begin{array}{l}-0.403124 \\
\pm 0.071\end{array}$ & $\begin{aligned} & 0.446949 \\
\pm & 1.48\end{aligned}$ \\
\hline$\frac{1}{4}$ & $\begin{array}{r}2.148757 \\
\pm 0.000027\end{array}$ & $\begin{aligned} & 3.084643 \\
\pm & 0.00011\end{aligned}$ & $\begin{aligned} & 3.262960 \\
\pm & 0.0013\end{aligned}$ & $\begin{array}{l}-0.405569 \\
\pm 0.023\end{array}$ & $\begin{aligned} & 0.396311 \\
\pm & 0.50\end{aligned}$ \\
\hline$\frac{1}{2}$ & $\begin{aligned} & 2.148743 \\
\pm & 0.00021\end{aligned}$ & $\begin{aligned} & 3.084617 \\
\pm & 0.00034\end{aligned}$ & $\begin{aligned} & 3.262768 \\
\pm & 0.0019\end{aligned}$ & $\begin{array}{l}-0.407940 \\
\pm 0.018\end{array}$ & $\begin{aligned} & 0.356202 \\
\pm & 0.21\end{aligned}$ \\
\hline$\frac{3}{4}$ & $\begin{aligned} & 2.148744 \\
\pm & 0.00014\end{aligned}$ & $\begin{aligned} & 3.084617 \\
\pm & 0.00025\end{aligned}$ & $\begin{aligned} & 3.262767 \\
\pm & 0.014\end{aligned}$ & $\begin{array}{l}-0.407989 \\
\pm 0.0019\end{array}$ & $\begin{aligned} & 0.355043 \\
\pm & 0.16\end{aligned}$ \\
\hline 1 & $\begin{aligned} & 2.148772 \\
\pm & 0.00039\end{aligned}$ & $\begin{aligned} & 3.0846503 \\
\pm & 0.00037\end{aligned}$ & $\begin{aligned} & 3.262906 \\
\pm & 0.0011\end{aligned}$ & $\begin{array}{l}-0.406974 \\
\pm 0.0056\end{array}$ & $\begin{aligned} & 0.365212 \\
\pm & 0.039\end{aligned}$ \\
\hline 2 & $\begin{aligned} & 2.148146 \\
\pm & 0.0087\end{aligned}$ & $\begin{aligned} & 3.084355 \\
\pm & 0.0040\end{aligned}$ & $\begin{aligned} & 3.262411 \\
\pm & 0.0069\end{aligned}$ & $\begin{array}{l}-0.408305 \\
\pm 0.019\end{array}$ & $\begin{aligned} & 0.361331 \\
\pm & 0.071\end{aligned}$ \\
\hline 3 & $\begin{aligned} & 2.296591 \\
\pm & 2.55\end{aligned}$ & $\begin{aligned} & 3.108788 \\
\pm & 0.42\end{aligned}$ & $\begin{aligned} & 3.277819 \\
\pm & 0.25\end{aligned}$ & $\begin{array}{l}-0.391079 \\
\pm 0.26\end{array}$ & $\begin{aligned} & 0.388505 \\
\pm & 0.38\end{aligned}$ \\
\hline
\end{tabular}

Table 1. Values obtained for $Z_{F, 10}\left(\frac{1}{2}+i T, \beta\right)$ using (4-8) with test function $g(s)=e^{-i \beta s}$, and using the known Satake parameters for $F$ for $p \leq 79$.

The results for $Z_{F, 10}\left(\frac{1}{2}+i\right)$ given by (4-5) and (4-6) are, respectively,

$$
3.084662 \pm 0.00047 \text { and } 3.084649 \pm 0.00056 \text {. }
$$

These values are consistent with each other. We view this as a confirmation of the conjectured functional equation for $L_{F, 10}$. In Theorem 4.2, we make precise what it means to provide confirmation of a conjectured functional equation.

We summarize the results of similar calculations, for various $s$ and functions $g$, in Table 1. Each column of the table corresponds to a value for $s$, and each row corresponds to a function $g(s)=e^{-i \beta s}$ in Theorem 4.1. Scanning down each column shows that the values are consistent, which gives a check on the functional equation for $L_{F, 10}$. We make this more precise in the next section.

Numerically checking the functional equation. We wish to check that the adjoint L-function given by the Euler product (2-1) has an analytic continuation which satisfies the functional equation. This requires that we evaluate the function outside the region where the Euler product converges, and also check that these values are consistent with the functional equation. In what follows we fix the genus 2 
Siegel modular form to be the unique weight 20 eigenform, $F$, that is not a SaitoKurokawa lift. Recall that we can use the calculations in [Kohnen and Kuss 2002] to determine the Satake parameters of $F$ for the primes $p \leq 79$.

Let $g(s)=g(s, \beta)=e^{-i \beta s}$ in Theorem 4.1. This meets the conditions of the theorem if $|\beta|<\frac{\pi}{4} \sum \kappa_{j}$. We use (4-3) to test the functional equation. This cannot be done in a naive way, because $\Lambda(s)$, as given by the right side of (4-3), automatically satisfies $\Lambda(s)=\varepsilon \bar{\Lambda}(1-s)$. Instead we exploit the fact that the right side (4-3), with our choice of $g$, has $\beta$ as a free parameter.

Rewrite (4-3) as $\Lambda(s)=g(s)^{-1} \Upsilon\left(s, Q, \kappa, \lambda, \varepsilon,\left\{b_{n}\right\}, \beta\right)$, and let

$$
L(s, \beta)=Q^{-s}\left(\prod_{j=1}^{a} \Gamma\left(\kappa_{j} s+\lambda_{j}\right)\right)^{-1} g(s, \beta)^{-1} \Upsilon\left(s, Q, \kappa, \lambda, \varepsilon,\left\{b_{n}\right\}, \beta\right) .
$$

Our test for the functional equation of $L(s)$ is that $L(s, \beta)$ is independent of $\beta$. That is, we check the consistency equation

$$
Z\left(s, \beta_{1}\right)-Z\left(s, \beta_{2}\right)=0 \text {. }
$$

For example, using (4-5) and (4-6) gives (4-9) in the form

$$
\begin{aligned}
& Z_{F, 10}\left(\frac{1}{2}+i, 0\right)-Z_{F, 10}\left(\frac{1}{2}+i, 3 / 2\right) \\
&= 0.150138-0.0998628 b_{2}-0.188349 b_{3}-0.20923 b_{4}-0.201655 b_{5} \\
& \quad+\cdots+4.80503 \times 10^{-7} b_{82}-8.76677 \times 10^{-7} b_{83}+\cdots \\
& \quad-1.57286 \times 10^{-6} b_{149}-1.49761 \times 10^{-6} b_{150}+\cdots \\
&= 0 .
\end{aligned}
$$

As described immediately before (4-7), we can estimate the contribution of the $b_{n}$ which are not known. The result is

$$
\begin{aligned}
& Z_{F, 10}\left(\frac{1}{2}+i, 0\right)-Z_{F, 10}\left(\frac{1}{2}+i, 3 / 2\right) \\
&= 0.150138-0.0998628 b_{2}-0.188349 b_{3}-0.20923 b_{4}-0.201655 b_{5} \\
&+\cdots+4.80503 \times 10^{-7} b_{82}+\cdots-1.49761 \times 10^{-6} b_{150}+\cdots \\
&= \Theta \times 0.00077,
\end{aligned}
$$

where $|\Theta| \leq 1$.

Now we can explain our method of evaluating the strength of (4-11) as a test of the conjectured functional equation. We wish to quantify the intuitive notion that it is unlikely for (4-11) to be true just by chance, because the coefficients of the $b_{n}$ are large compared to the right side of the equation. We do this by considering the $b_{n}$ to be random variables, and furthermore we make some assumptions about their 
probability density functions. This, of course, requires some justification which we now provide.

L-functions naturally fall into families [Conrey et al. 2005; Katz and Sarnak 1999], and the collection of L-functions in a family can be modeled statistically. For example, for the family of GL(2) L-functions, each coefficient $b_{j}$ has a particular distribution. The distribution of $b_{p}$, for $p$ prime, tends to the Sato-Tate distribution as $p \rightarrow \infty$, and $b_{n}, b_{m}$ are uncorrelated if $(n, m)=1$. See [Conrey et al. 1997; Serre 1997] for details.

For other families, there are other distributions, see [Kedlaya and Sutherland 2009] for several examples. These distributions are the distributions of traces of matrices in a compact group, weighted according to Haar measure. For the Siegel modular forms we consider here, the Hecke eigenvalues are expected to be distributed according to an $\operatorname{Sp}(4, \mathbb{Z})$ analogue of the $\mathrm{GL}(2)$ case. This leads to a conjecture for the distribution of the Dirichlet series coefficients of the degree-10 L-function we are considering here. (See additional comments at the end of this section.)

Thus, over the family of L-functions associated to Siegel modular forms, we assume the $b_{p}$ behave as independent random variables, each of which has a continuous probability distribution which is supported on $[-10,10]$ and which is bounded by 1 , say. If we focus on one coefficient, say $b_{3}$, and first choose all the other $b_{n}$, then (4-11) becomes

$$
C-0.188349 b_{3}=\Theta \times 0.00077,
$$

where $C$ is some number. Hence, there is a $C^{\prime}$ so that,

$$
b_{3} \in\left[C^{\prime}-0.004088, C^{\prime}+0.004088\right] \text {. }
$$

Since the PDF of $b_{3}$ is assumed to be bounded by 1 , the probability of (4-13) being true is less than the length of that interval, which is 0.00817 . In other words, there is less than a 1 percent chance that $L_{F, 10}$ would accidentally pass that test for satisfying the functional equation. We have proven:

Theorem 4.2. Fix the parameters in the functional equation of $L(s)$, as described on page 359, and suppose the coefficients $b_{j}, j \in J$, are known, while the remaining coefficients obey the Ramanujan bound. Let $L(s, \beta)$ be given by (4-8), and choose real numbers $\beta_{1}, \beta_{2}$ and a complex number $s_{0}$. Write

$$
Z\left(s_{0}, \beta_{1}\right)-Z\left(s_{0}, \beta_{2}\right)=\sum_{j} v_{j} b_{j}=\sum_{j \in J} v_{j} b_{j}+\Theta \delta,
$$

where $|\Theta|<1$ and $\delta$ is determined as described in page 364 . 
If the $b_{j}$ for $j \in J$ are chosen independently from continuous probability distributions whose PDFs are bounded by 1, then the probability that (4-14) is consistent with the functional equation is less than $\delta /\left|v_{j}\right|$ for any $j \in J$.

It is easy to extend Theorem 4.2 to the case of several equations. Suppose we know $b_{j}$ for $j \in J$, and choose $s_{k}, \beta_{k, 1}$, and $\beta_{k, 2}$ for $1 \leq k \leq K$. We have

$$
Z\left(s_{k}, \beta_{k, 1}\right)-Z\left(s_{k}, \beta_{k, 2}\right)=\sum_{j} v_{k, j} b_{j}=\sum_{j \in J} v_{k, j} b_{j}+\Theta_{k} \delta_{k} .
$$

Select $b_{j_{1}}, \ldots, b_{j_{K}}$, and suppose all the other $b_{j}$ have been determined. Then the system

$$
\left\{Z\left(s_{k}, \beta_{k, 1}\right)-Z\left(s_{k}, \beta_{k, 2}\right)=0\right\}_{k=1}^{K}
$$

is equivalent to

$$
A\left(b_{j_{1}}, \ldots, b_{j_{K}}\right) \in\left(C_{1}, \ldots, C_{K}\right)+\left[-\delta_{1}, \delta_{1}\right] \times \cdots \times\left[-\delta_{K}, \delta_{K}\right],
$$

where $A$ is the matrix $\left(v_{k, j_{k}}\right)$. So we can rewrite the condition on the $b_{j_{k}}$ as

$$
\left(b_{j_{1}}, \ldots, b_{j_{K}}\right) \in A^{-1}\left(C_{1}, \ldots, C_{K}\right)+A^{-1}\left(\left[-\delta_{1}, \delta_{1}\right] \times \cdots \times\left[-\delta_{K}, \delta_{K}\right]\right) .
$$

Since the PDFs of $b_{n_{j}}$ are assumed to be bounded by 1, the probability that (4-18) occurs is bounded by the volume of the right side, which is $2^{K}|\operatorname{det} A|^{-1} \delta_{1} \cdots \delta_{K}$.

Here is an example using some of the data from Table 1. Pairing the first and fifth entries of column 2, and the fifth and sixth entries in column 3 , we find

$$
\text { (4-19) } \begin{array}{r}
Z\left(\frac{1}{2}+i, 0\right)-Z\left(\frac{1}{2}+i, 1\right) \\
=-0.07393+0.05869 b_{2}+0.10175 b_{3}+\cdots \pm 0.00013, \\
\text { (4-20) } \begin{array}{r}
Z\left(\frac{1}{2}+2 i, 1\right)-Z\left(\frac{1}{2}+2 i, 2\right) \\
=-0.41376+0.18021 b_{2}+0.43401 b_{3}+\cdots \pm 0.0077 .
\end{array}
\end{array}
$$

Using the coefficients of $b_{2}$ and $b_{3}$ in (4-18) we find that the probability of the above system being satisfied for random $b_{2}, b_{3}$ is less than 0.00059 . This strikes us as rather convincing evidence that the expected functional equation of $L_{F, 10}$ is in fact correct.

We have a few comments on these calculations. Our purpose is to show that it is possible to quantify the precision to which changes to the test function in the approximate functional equation give a check on the functional equation of an L-function. Since it is, in a sense, nonsensical to treat the known coefficients of an L-function as random, we have tried not to push the analogy too far. If the coefficients $b_{j}$ really were independent and random, then the sum involving $b_{j}$ in equations like (4-11) would have a very large variance and the probability that (4-11) holds would be much smaller than our estimate. We chose to focus on just 
one or two coefficients at a time in order to not stretch plausibility too much. Also, the method of (4-18) gives poor results if the matrix is close to singular. In fact, one can add a new equation and increase the probability that the system is consistent, which is absurd. This can happen in practice: adding another equation based on Table 1 to (4-19) and (4-20) actually gives worse results. This is due to a dependence among the equations, arising from the fact that for small $t$ it takes relatively few coefficients to evaluate $L\left(\frac{1}{2}+i t\right)$. We will return to this topic elsewhere.

Rigorously evaluating L-functions. Earlier (page 359) we estimated the contribution of the terms involving the $b_{n}$ which were not known explicitly, but only assumed to satisfy the Ramanujan bound. This involves estimating the contribution of infinitely many terms and occurs in two steps. First, using Lemma 4.3 below, we determine $N$ and $\delta_{1}$ so that the terms involving $b_{n}$ with $n>N$ contribute, in total, less than $\delta_{1}$. Then we explicitly evaluate the contributions of the terms $f_{1}(s, n)$ and $f_{2}(s, n)$ occurring in (4-3) for $83 \leq n \leq N$; call that contribution $\delta_{2}$. Then our estimate for the contribution of the unknown terms is $\delta_{1}+\delta_{2}$.

For example, let $\beta=1$ and $s=\frac{1}{2}+i$, in order to obtain the entry in the fifth row and second column of Table 1 . With $N=10,000$ we find $\delta_{1}<10^{-6}$ and $\delta_{2}<0.000373$, as reported. This approach was used to determine the values in Table 1 and elsewhere in Section 4.

The following is a very slight modification of [Booker 2006, Lemma 5.2].

\section{Lemma 4.3. Let}

$$
G^{*}\left(u ; \eta,\left\{\mu_{j}\right\}\right):=\frac{1}{2 \pi i} \int_{\nu} e^{\left(u+i \frac{\pi \tau}{4} \eta\right)\left(\frac{1}{2}-s\right)} \prod_{j=1}^{r} \Gamma_{\mathbb{R}}\left(s+\mu_{j}\right) \frac{d s}{s} .
$$

Then, for $X \geq r$,

$$
G^{*}\left(u ; \eta,\left\{\mu_{j}\right\}\right) \leq \frac{K r}{X} e^{\Re(\mu) u} e^{-X} \prod_{j=1}^{r}\left(1+\frac{r v_{j}}{X}\right)^{v_{j}},
$$

where $\delta=\frac{\pi}{2}(1-|\eta|), v_{v}=\frac{1}{2}\left(\Re \mu_{j}-1\right), \mu=\frac{1}{2}+\frac{1}{r}\left(1+\sum \mu_{j}\right), X=\pi r \delta e^{-\delta} e^{2 u / r}$, and

$$
K=2 \sqrt{\frac{2^{r+1}}{r} \frac{e^{\delta(r-1)}}{\delta}} e^{-\pi r \eta \Im \mu / 4} .
$$

Note that our $G^{*}$ is identical to Booker's function $G$ except for the extra factor of $1 / s$ in the integrand.

Proof. Move the line of integration to the $2 \sigma$ line and let $s=2 \sigma+2 i t$ and use the trivial estimate $1 /|s| \leq 1 /(2 \sigma)$ to get

$$
G^{*}\left(u ; \eta,\left\{\mu_{j}\right\}\right) \leq \frac{1}{\sigma} \frac{1}{2 \pi} \int_{2 \sigma}\left|e^{(u+i \pi \tau \eta / 4)(1 / 2-s)} \prod_{j=1}^{r} \Gamma_{\mathbb{R}}\left(s+\mu_{j}\right)\right| d t .
$$


Now exactly follow the proof of Lemma 5.2 in [Booker 2006], which in the last step chooses $\sigma=X / r$.

\section{Acknowledgement}

We thank Sharon Garthwaite for her helpful comments on the paper and the referee for helping improve the paper.

\section{References}

[Andrianov 1974] A. N. Andrianov, "Euler products corresponding to Siegel modular forms of genus 2", Uspehi Mat. Nauk 29:3 (177) (1974), 43-110. In Russian; translated in Russ. Math. Surv. 29:3 (1974), 45-116. MR 55 \#5540 Zbl 0304.10021

[Asgari and Schmidt 2001] M. Asgari and R. Schmidt, "Siegel modular forms and representations", Manuscripta Math. 104:2 (2001), 173-200. MR 2002a:11044 Zbl 0987.11037

[Böcherer 1985] S. Böcherer, "Über die Funktionalgleichung automorpher $L$-Funktionen zur Siegelschen Modulgruppe”, J. Reine Angew. Math. 362 (1985), 146-168. MR 87h:11039 Zbl 0565.10025

[Booker 2006] A. R. Booker, "Artin's conjecture, Turing's method, and the Riemann hypothesis", Experiment. Math. 15:4 (2006), 385-407. MR 2007k:11084 Zbl 1169.11019

[Borel 1979] A. Borel, "Automorphic L-functions", pp. 27-61 in Automorphic forms, representations and L-functions (Corvallis, OR, 1977), vol. 2, edited by A. Borel and W. Casselman, Proc. Sympos. Pure Math. 33, Amer. Math. Soc., Providence, R.I., 1979. MR 81m:10056 Zbl 0412.10017

[Conrey et al. 1997] J. B. Conrey, W. Duke, and D. W. Farmer, "The distribution of the eigenvalues of Hecke operators”, Acta Arith. 78:4 (1997), 405-409. MR 98k:11047 Zbl 0876.11020

[Conrey et al. 2005] J. B. Conrey, D. W. Farmer, J. P. Keating, M. O. Rubinstein, and N. C. Snaith, "Integral moments of L-functions", Proc. London Math. Soc. (3) 91:1 (2005), 33-104. MR 2006j:11120 Zbl 1075.11058

[Farmer et al. $\geq 2011]$ D. W. Farmer, S. Koutsoliotas, and S. Lemurell, "Maass forms on GL(3) and GL(4)", preprint.

[Gan and Takeda 2010] W. T. Gan and S. Takeda, "The local Langlands conjecture for Sp(4)", Int. Math. Res. Not. 2010:15 (2010), 2987-3038. MR 2011g:22027 Zbl 05786178

[Gelbart 1984] S. Gelbart, "An elementary introduction to the Langlands program", Bull. Amer. Math. Soc. (N.S.) 10:2 (1984), 177-219. MR 85e:11094 Zbl 0539.12008

[Ginzburg 1996] D. Ginzburg, "A Rankin-Selberg integral for the adjoint $L$-function of $\mathrm{Sp}_{4}$ ", Israel J. Math. 95 (1996), 301-339. MR 97k:11081 Zbl 0869.11046

[Katz and Sarnak 1999] N. M. Katz and P. Sarnak, Random matrices, Frobenius eigenvalues, and monodromy, American Mathematical Society Colloquium Publications 45, American Mathematical Society, Providence, RI, 1999. MR 2000b:11070 Zbl 0958.11004

[Kedlaya and Sutherland 2009] K. S. Kedlaya and A. V. Sutherland, "Hyperelliptic curves, Lpolynomials, and random matrices", pp. 119-162 in Arithmetic, geometry, cryptography and coding theory (Marseilles, 2007), edited by G. Lachaud et al., Contemp. Math. 487, Amer. Math. Soc., Providence, RI, 2009. MR 2011d:11154 Zbl 05592921

[Knapp 1994] A. W. Knapp, "Local Langlands correspondence: the Archimedean case", pp. 393-410 in Motives (Seattle, 1991), edited by U. Jannsen et al., Proc. Sympos. Pure Math. 55, Amer. Math. Soc., Providence, RI, 1994. MR 95d:11066 Zbl 0811.11071 
[Kohnen and Kuss 2002] W. Kohnen and M. Kuss, "Some numerical computations concerning spinor zeta functions in genus 2 at the central point", Math. Comp. 71:240 (2002), 1597-1607. MR 2003i:11057 Zbl 1076.11030

[Roberts and Schmidt 2007] B. Roberts and R. Schmidt, Local newforms for GSp(4), Lecture Notes in Mathematics 1918, Springer, Berlin, 2007. MR 2008g:11080 Zbl 1126.11027

[Rubinstein 2005] M. Rubinstein, "Computational methods and experiments in analytic number theory", pp. 425-506 in Recent perspectives in random matrix theory and number theory, edited by F. Mezzadri and N. C. Snaith, London Math. Soc. Lecture Note Ser. 322, Cambridge Univ. Press, Cambridge, 2005. MR 2006d:11153 Zbl 1168.11329

[Ryan and Shemanske 2008] N. C. Ryan and T. R. Shemanske, "Inverting the Satake map for $\mathrm{Sp}_{n}$ and applications to Hecke operators”, Ramanujan J. 17:2 (2008), 219-244. MR 2009i:11064 Zbl 1213.11108

[Selberg 1992] A. Selberg, "Old and new conjectures and results about a class of Dirichlet series", pp. 367-385 in Proceedings of the Amalfi Conference on Analytic Number Theory (Maiori, Italy, 1989), edited by E. Bombieri et al., Univ. Salerno, Salerno, 1992. MR 94f:11085 Zbl 0787.11037

[Serre 1997] J.-P. Serre, "Répartition asymptotique des valeurs propres de l'opérateur de Hecke $T_{p}$ ", J. Amer. Math. Soc. 10:1 (1997), 75-102. MR 97h:11048 Zbl 0871.11032

[Shimura 1994] G. Shimura, "Euler products and Fourier coefficients of automorphic forms on symplectic groups”, Invent. Math. 116:1-3 (1994), 531-576. MR 96f:11065 Zbl 0811.11033

[Skoruppa 1992] N.-P. Skoruppa, "Computations of Siegel modular forms of genus two", Math. Comp. 58:197 (1992), 381-398. MR 92e:11041 Zbl 0749.11030

[Tate 1979] J. Tate, "Number theoretic background", pp. 3-26 in Automorphic forms, representations and L-functions (Corvallis, OR, 1977), vol. 2, edited by A. Borel and W. Casselman, Proc. Sympos. Pure Math. 33, Amer. Math. Soc., Providence, R.I., 1979. MR 80m:12009 Zbl 0422.12007

[Weissauer 2009] R. Weissauer, Endoscopy for GSp(4) and the cohomology of Siegel modular threefolds, Lecture Notes in Mathematics 1968, Springer, Berlin, 2009. MR 2010h:11086 Zbl 05490504

Received October 13, 2008. Revised September 10, 2010.

DAVID W. FARMER

AMERICAN InSTITUTE OF MATHEMATICS

Palo Alto, CA 94306

UNITED STATES

farmer@aimath.org

NATHAN C. RYAN

DEPARTMENT OF MATHEMATICS

BUCKNELL UNIVERSITY

LEWISBURG, PA 17837

UNITED STATES

nathan.ryan@bucknell.edu

RALF SCHMIDT

DEPARTMENT OF MATHEMATICS

UNIVERSITY OF OKLAHOMA

NORMAN, OK 73072

United STATES

rschmidt@math.ou.edu 


\title{
PACIFIC JOURNAL OF MATHEMATICS
}

\author{
http://pacificmath.org \\ Founded in 1951 by \\ E. F. Beckenbach (1906-1982) and F. Wolf (1904-1989)
}

\section{EDITORS}

V. S. Varadarajan (Managing Editor)

Department of Mathematics

University of California

Los Angeles, CA 90095-1555

pacific@math.ucla.edu

Vyjayanthi Chari

Department of Mathematics

University of California

Riverside, CA 92521-0135

chari@math.ucr.edu

\section{Robert Finn}

Department of Mathematics Stanford University

Stanford, CA 94305-2125

finn@math.stanford.edu

Kefeng Liu

Department of Mathematics

University of California

Los Angeles, CA 90095-1555

liu@math.ucla.edu
Darren Long

Department of Mathematics

University of California

Santa Barbara, CA 93106-3080

long@math.ucsb.edu

Jiang-Hua Lu

Department of Mathematics

The University of Hong Kong

Pokfulam Rd., Hong Kong jhlu@maths.hku.hk

Alexander Merkurjev

Department of Mathematics

University of California

Los Angeles, CA 90095-1555

merkurev@math.ucla.edu
Sorin Popa

Department of Mathematics University of California

Los Angeles, CA 90095-1555 popa@math.ucla.edu

Jie Qing

Department of Mathematics

University of California

Santa Cruz, CA 95064

qing@cats.ucsc.edu

Jonathan Rogawski

Department of Mathematics

University of California

Los Angeles, CA 90095-1555

jonr@math.ucla.edu

\section{PRODUCTION}

pacific@math.berkeley.edu

\section{SUPPORTING INSTITUTIONS}

ACADEMIA SINICA, TAIPEI

CALIFORNIA INST. OF TECHNOLOGY INST. DE MATEMÁTICA PURA E APLICADA KEIO UNIVERSITY

MATH. SCIENCES RESEARCH INSTITUTE NEW MEXICO STATE UNIV.

OREGON STATE UNIV.

\author{
STANFORD UNIVERSITY \\ UNIV. OF BRITISH COLUMBIA \\ UNIV. OF CALIFORNIA, BERKELEY \\ UNIV. OF CALIFORNIA, DAVIS \\ UNIV. OF CALIFORNIA, LOS ANGELES \\ UNIV. OF CALIFORNIA, RIVERSIDE \\ UNIV. OF CALIFORNIA, SAN DIEGO \\ UNIV. OF CALIF., SANTA BARBARA
}

\author{
UNIV. OF CALIF., SANTA CRUZ \\ UNIV. OF MONTANA \\ UNIV. OF OREGON \\ UNIV. OF SOUTHERN CALIFORNIA \\ UNIV. OF UTAH \\ UNIV. OF WASHINGTON \\ WASHINGTON STATE UNIVERSITY
}

These supporting institutions contribute to the cost of publication of this Journal, but they are not owners or publishers and have no responsibility for its contents or policies.

See inside back cover or pacificmath.org for submission instructions.

The subscription price for 2011 is US \$420/year for the electronic version, and \$485/year for print and electronic.

Subscriptions, requests for back issues from the last three years and changes of subscribers address should be sent to Pacific Journal of Mathematics, P.O. Box 4163, Berkeley, CA 94704-0163, U.S.A. Prior back issues are obtainable from Periodicals Service Company, 11 Main Street, Germantown, NY 12526-5635. The Pacific Journal of Mathematics is indexed by Mathematical Reviews, Zentralblatt MATH, PASCAL CNRS Index, Referativnyi Zhurnal, Current Mathematical Publications and the Science Citation Index.

The Pacific Journal of Mathematics (ISSN 0030-8730) at the University of California, c/o Department of Mathematics, 969 Evans Hall, Berkeley, CA 94720-3840, is published monthly except July and August. Periodical rate postage paid at Berkeley, CA 94704, and additional mailing offices. POSTMASTER: send address changes to Pacific Journal of Mathematics, P.O. Box 4163, Berkeley, CA 94704-0163.

PJM peer review and production are managed by EditFLOW ${ }^{\mathrm{TM}}$ from Mathematical Sciences Publishers.

PUBLISHED BY PACIFIC JOURNAL OF MATHEMATICS

at the University of California, Berkeley 94720-3840

A NON-PROFIT CORPORATION

Typeset in LATEX

Copyright $(2011$ by Pacific Journal of Mathematics 


\section{PACIFIC JOURNAL OF MATHEMATICS}

Volume $253 \quad$ No. $2 \quad$ October 2011

Fusion rules on a parametrized series of graphs

MARTA AsAeda and UfFE HAAGERUP

Group gradings on restricted Cartan-type Lie algebras

YURI BAHTURIN and MIKHAIL KOCHETOV

B2-convexity implies strong and weak lower semicontinuity of partitions of $\mathbb{R}^{n}$

\section{DAVID G. CARABALLO}

Testing the functional equation of a high-degree Euler product

DAVID W. FARMER, NATHAN C. RYAN and RALF SCHMIDT

Asymptotic structure of a Leray solution to the Navier-Stokes flow around a 367 rotating body

Reinhard Farwig, Giovanni P. Galdi and Mads Kyed

Type II almost-homogeneous manifolds of cohomogeneity one

DANIEL GUAN

Cell decompositions of Teichmüller spaces of surfaces with boundary

REN GUO and FENG LUO

A system of third-order differential operators conformally invariant under $\mathfrak{s l}(3, \mathbb{C})$ and $\mathfrak{s o}(8, \mathbb{C})$

Toshinis A KUBO

Axial symmetry and regularity of solutions to an integral equation in a half-space

GUOZHEN LU and JIUYi ZHU

Braiding knots in contact 3-manifolds

\section{ELENA PAVELESCU}

Gradient estimates for positive solutions of the heat equation under geometric flow

JUN SUN 